\title{
Short communication: Mouse mammary tumor virus driven a-lactalbumin expression effects on lactation and fertility of transgenic mice
}

\author{
S. Le Guillou, ${ }^{1}$ G. Tilly, B. Passet, L. Lefèvre, M. Vilotte, J. Costa, F. Le Provost, and J.-L. Vilotte \\ INRA, UMR 1313 Génétique Animale et Biologie Intégrative, Bâtiment 440, F-78350 Jouy-en-Josas, France
}

\begin{abstract}
$\alpha$-Lactalbumin (Alac) is one of the major milk proteins. Its gene expression is restricted to epithelial cells of the lactating mammary gland. The Alac interaction with a uridine 5'-diphosphate-galactosyltransferase induces lactose synthesis, a major osmotic regulator of milk secretion. Other functions attributed to this protein include induction of apoptosis and anti-inflammatory activities. To assess if forced expression of this gene during early gestation or involution could affect mammary physiology, an Alac-encoding minigene was expressed in transgenic mice under the transcriptional regulation of the mouse mammary tumor virus promoter. The mammary expression did not interfere with gestation, resulted in a slight increase in milk yield as indirectly assessed by the $11 \%$ increased growth rate of the pups reared by transgenic females compared with that of those reared by control mice, and induced a slight delay in the early involution process, as demonstrated by histological analyses. The use of the mouse mammary tumor virus promoter resulted in Alac expression in several nonmammary tissues, such as the brain, the testis, the ovary, and the uterus. Although it did not affect male reproductive performances, it induced a female subfertile phenotype, characterized by embryonic implantation failure in the transgenic female reproductive tract.
\end{abstract}

Key words: $\alpha$-lactalbumin, mammary gland, fertility, transgenic mouse

\section{Short Communication}

The calcium metalloprotein $\alpha$-lactalbumin (Alac) is a major whey protein found in the milk of most mammalian species. It complexes with the golgi uridine 5 '-diphosphate (UDP)-galactosyltransferase (EC 2.4.1.22) to form the lactose synthase complex. This enzyme is responsible for the production of lactose, a key osmotic regulator of milk secretion (Noble et al.,

Received November 24, 2010.

Accepted January 31, 2011.

${ }^{1}$ Corresponding author: sandrine.leguillou@jouy.inra.fr
2002). The expression of the Alac gene is restricted to the lactating mammary epithelial cells. The level of Alac increases near parturition, remains elevated and constant during lactation, and decreases sharply at dry-off and during involution. The protein profile follows a similar trend (Bleck and Bremel, 1994). Overexpression of Alac in lactating mammary glands was reported in transgenic mice (Vilotte et al., 1989; Bleck and Bremel, 1994; Stacey et al., 1995) and cow (Wang et al., 2008). The recombinant protein induced lactose synthesis, even when originating from a different species than the host (Stacey et al., 1995; Soulier et al., 1997; Bleck et al., 1998), and increased milk yield or growth of the pups or both (Boston et al., 2001; Wheeler et al., 2001). Deletion of this gene in transgenic mice resulted in the synthesis of a highly viscous, lactose-free milk that pups were unable to suckle (Stinnakre et al., 1994; Stacey et al., 1995).

Beside its role in promoting lactose synthesis, other functions have been attributed to Alac, such as a cell lytic activity (McKenzie and White, 1987) and induction of cell growth inhibition (Thompson et al., 1992). A folding variant of Alac, complexed with oleic acid, was shown to mediate apoptotic cell death in vitro (Hakansson et al., 1995; Svensson et al., 2003a,b). The broad, but yet selective, specificity of these complexes suggested that Alac might not only regulate the function of lymphocytes and epithelium of the new-born through milk consumption (Hakansson et al., 1995), but also participate in the control of mammary epithelial cell apoptosis during involution (Baltzer et al., 2004; Riley et al., 2008).

So far, Alac from various species were overexpressed only specifically in the lactating mammary gland of transgenic mammals using Alac-encoding gene regulatory sequences or, in one case, the as1-casein promoter (Fujiwara et al., 1999). To assess if forced expression of this gene during early gestation or involution could affect mammary physiology, potentially highlighting the significance of some of the noncanonical functions attributed to Alac, a goat Alac-encoding minigene was expressed in transgenic mice under the transcriptional regulation of the mouse mammary tumor virus (MMTV) promoter derived from the pMSG expres- 
A
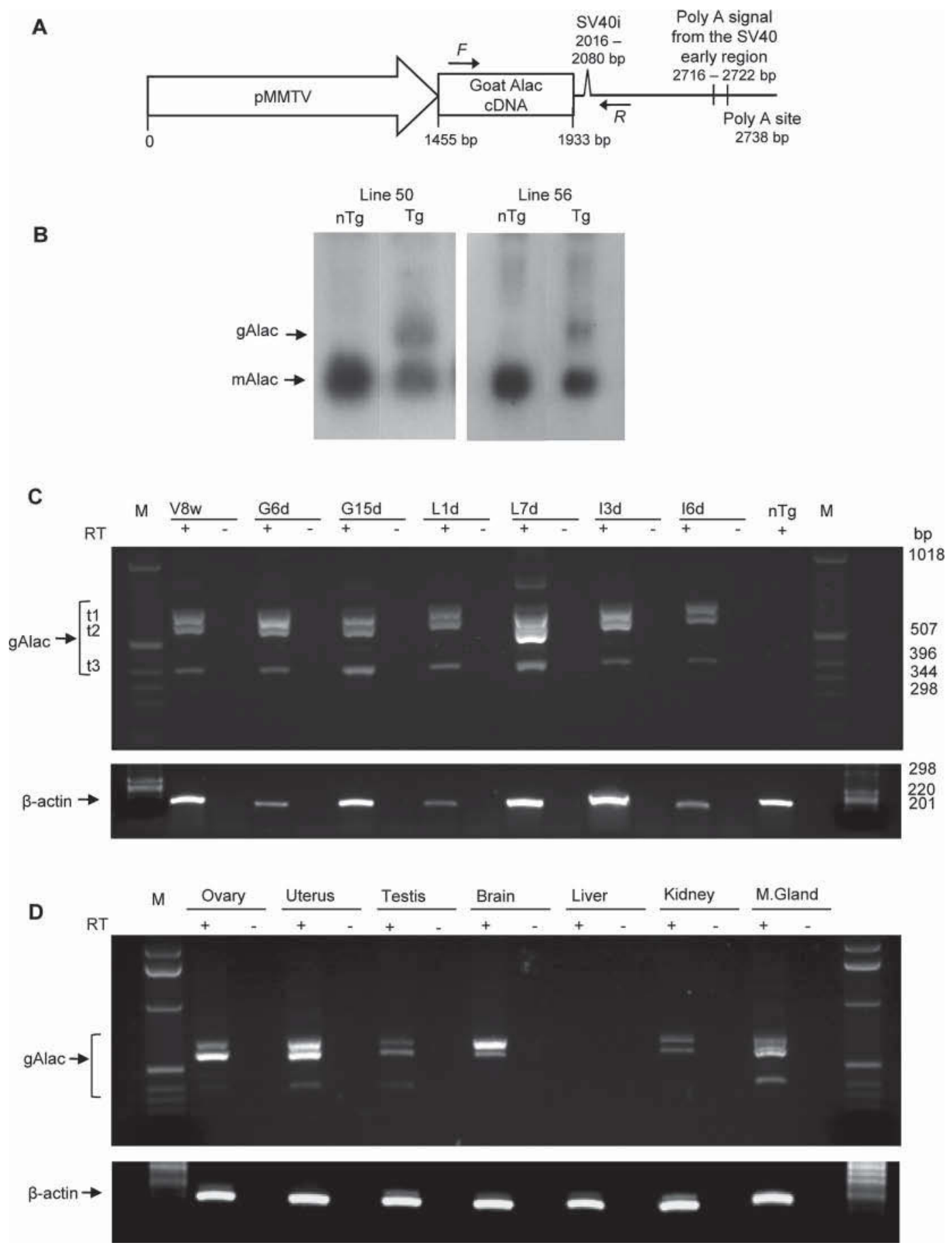

Figure 1. Goat $\alpha$-lactalbumin (gAlac) transgene expression in the mammary gland. (A) Schematic representation of the goat Alac minigene construction, 3,150 bp. pMMTV = mouse mammary tumor virus promoter; goat Alac $=$ gAlac open reading frame; SV40i $=$ SV40 small $\mathrm{T}$ antigen intron. The figure is not to scale. $F, R=$ positions of the forward and reverse gAlac primers used in reverse transcriptase (RT)-PCR analyses. (B) Northern analyses of the expression of the gAlac transgene in mammary gland of mice from lines 50 and 56 , at 7 and $2 \mathrm{~d}$ of lactation, respectively. $\mathrm{mAlac}=$ mouse Alac $\mathrm{mRNA} ; \mathrm{gAlac}=$ goat Alac-encoding transcript; $\mathrm{nTg}=$ nontransgenic mouse; $\mathrm{Tg}=$ transgenic mouse (C, D) RT-PCR analyses of the expression of gAlac transgene and mouse $\beta$-actin in mammary glands of line 50, at different stages of development (C) and in various tissues (D). V8w = 8-wk-old virgin; G6d = gestation d 6; G15d = gestation $\mathrm{d} 15 ; \mathrm{L} 1 \mathrm{~d}=$ lactation $\mathrm{d} 1 ;$ L7d $=$ lactation $\mathrm{d} 7 ; \mathrm{I} 3 \mathrm{~d}=$ post-weaning $\mathrm{d} 3$; I6d $=$ post-weaning $\mathrm{d} 6 ; \mathrm{nTg}=$ nontransgenic mammary gland at lactation $\mathrm{d} 10 ; \mathrm{M}$.Gland $=$ mammary gland at lactation d 1; $\mathrm{M}=1$-kb DNA ladder, Invitrogen (Cergy Pontoise, France); $\mathrm{t} 1=$ unspliced transgene-derived transcript; $\mathrm{t} 2=\mathrm{spliced}$ transcript; $\mathrm{t} 3=$ unexpected shorter transcript; $+=\mathrm{RT}-\mathrm{PCR}$ with addition of reverse transcriptase; $-=$ PCR on product of reverse transcriptase reaction lacking reverse transcriptase enzyme, as negative control. No amplification was observed in control tissues derived from nontransgenic mice (Figure 1C). In ovary and kidney, overexposure of the gel allows visualization of the t3 transcript (data not shown). 
sion vector (Amersham Pharmacia Biotech Inc., Orsay, France, and Figure 1A).

All animal manipulations were performed following the French Commission of Génie Génétique recommendations. The gel-purified construct was injected into the fertilized eggs of FVB/N mice. Six transgenic mice were identified out of the 67 resulting pups by PCR analysis of their tail genomic DNA using the set of primers 5'-ACCAGGGCCCTCACTAA- ${ }^{\prime}$ and 5'-GGAAAGTCCTTGGGGTCTTCTACC-3', the GoTaq Fexi DNA polymerase kit (Promega, Charbonnières-les-Bains, France), a hybridization temperature of $55^{\circ} \mathrm{C}$, an elongation time of $30 \mathrm{~s}$, and 40 amplification cycles. These 3 females and 3 males transmitted the transgene to their progeny. Northern expression analysis of the transgene in the mammary gland of lactating females from each line demonstrated that only 2 expressed detectable levels, lines 50 and 56 (Figure 1B and data not shown). The SYBR Green quantitative PCR (SYBR GREEN PCR Master Mix, Applied Biosystems, France) performed on genomic DNA, using a transgene-specific set of primers, 5'-TCTCTGCTCCTGGTAGGCAT-3' and 5'-TCAGGCAAACTGACACCTCC-3'PCR, allowed estimates showing that lines 50 and 56 carried 3 and 4 integrated copies of the transgene, respectively. This estimation was based on the result of the amplification of the $\beta 2$-microglobulin gene that was used as an internal control for the amount of genomic DNA using the set of primers, 5'-GTGACGACCTCCGGATCTGA-3' and 5'-GCCGAGTAGCAGCCACTGAA-3'. In both lines, integrated copies of the transgene were transmitted as a single locus, suggesting co-integration.

Further analysis of the transgene expression was performed in the mammary gland of animals from lines 50 and 56 by reverse transcriptase (RT)-PCR at various developmental stages. Total RNA were isolated from various tissues. The RQ1 RNase-free DNase treatment was performed according to Promega recommendations. The RT was performed using the SuperScript FirstStrand synthesis kit (Invitrogen, Cergy Pontoise, France) using the provided random primers. The PCR analyses were carried out with the following set of primers: goat transgene, 5'-GTTTGCCTGAATGGGTCTGT-3' and 5'-GGAAAGTCCTTGGGGTCTTC-3', and $\beta$-actin, 5-CACCAGTTCGCCATGGATG-3' and 5'-TCCCACCATCACACCCTG-3'. The transgene expression was detected at all studied stages (8-wk-old virgin, gestation d 6 and 15, lactation d 1 and 7, post-weaning d 3 and 6), as expected with a MMTV promoter (Figure 1C). During lactation, the transgene level appeared to be similar between the 2 lines, as assessed by RT-quantitative (q)PCR, and close, although slightly lower, to that of the endogenous gene (Figure 1B). The RT-PCR revealed the presence in all of the analyzed samples of
3 transcripts (named t1, t2, and $\mathrm{t} 3$ in Figure $1 \mathrm{C}$ and Figure 2) that resulted from alternative splicing events. The longest one, t1, corresponded to the unspliced transcript. The DNase treatment of the RNA samples before the reverse transcription reaction clearly indicated that it did not result from DNA contamination (Figure 1C). The second transcript, t2, corresponded to the expected spliced mRNA. These 2 RNA were the most highly expressed. The shorter one, $\mathrm{t} 3$, was characterized by an unexpected splicing event (Figure 2A). The $5^{\prime}$ donor-splicing site was located within the goat Alac open reading frame (gAlac ORF), the $3^{\prime}$ acceptor splicing site was the one present within the SV40 sequence. This latter mRNA encodes for a C-terminally extended matured protein of 281 AA that shared $100 \%$ homology with the first $98 \mathrm{AA}$ of the N-terminal region of Alac (Figure 2B). Western analysis of milk proteins using a polyclonal Alac-antibody (Vilotte et al., 1989) only revealed a band of the expected size in the milk of the transgenic mice (data not shown). It suggested that either 1) the polyclonal antibody does not recognize the C-terminally extended protein or more likely, 2) this protein is absent or only present at low levels in the milk.

Transgenic lactating females from both lines were able to sustain lactation. No significant difference was detected in the mean weights of litters between the transgenic lines and control mice at parturition (1.4 g $\pm 0.1 \mathrm{~g}$ per pup). Pups reared by transgenic dams grew faster $(+11 \%)$ than pups reared by control dams, as assessed by comparing 2 lactating transgenic mice with 2 control nontransgenic animals and after standardizing litter sizes to 8 pups per female. Such a phenotype was expected, as it was reported for transgenics overexpressing the Alac in their milk and attributed to an increase in milk production (Boston et al., 2001; Wheeler et al., 2001). Because in our model, Alac was present during all of the gestation period and maintained during involution, the structure of the mammary tissue was compared between transgenic tissues from both lines and control mouse mammary tissues at various developmental stages. No difference was visually apparent by whole mount and histological analyses at gestation d 6 and 15, performed as described previously (http://www.bcm.edu/rosenlab/ protocols/HEstaining.pdf), suggesting that gAlac expression did not interfere with mammary epithelial cell proliferation and differentiation during early gestation. It might result either from an unlikely inability of gAlac to associate in vivo with mouse fatty acids to form bovine $\alpha$-lactalbumin made lethal to tumor cells (BAMLET)-like complexes or, more likely, from the absence of the fatty acid partner of Alac, because the rate of fatty acid synthesis increases sharply around 


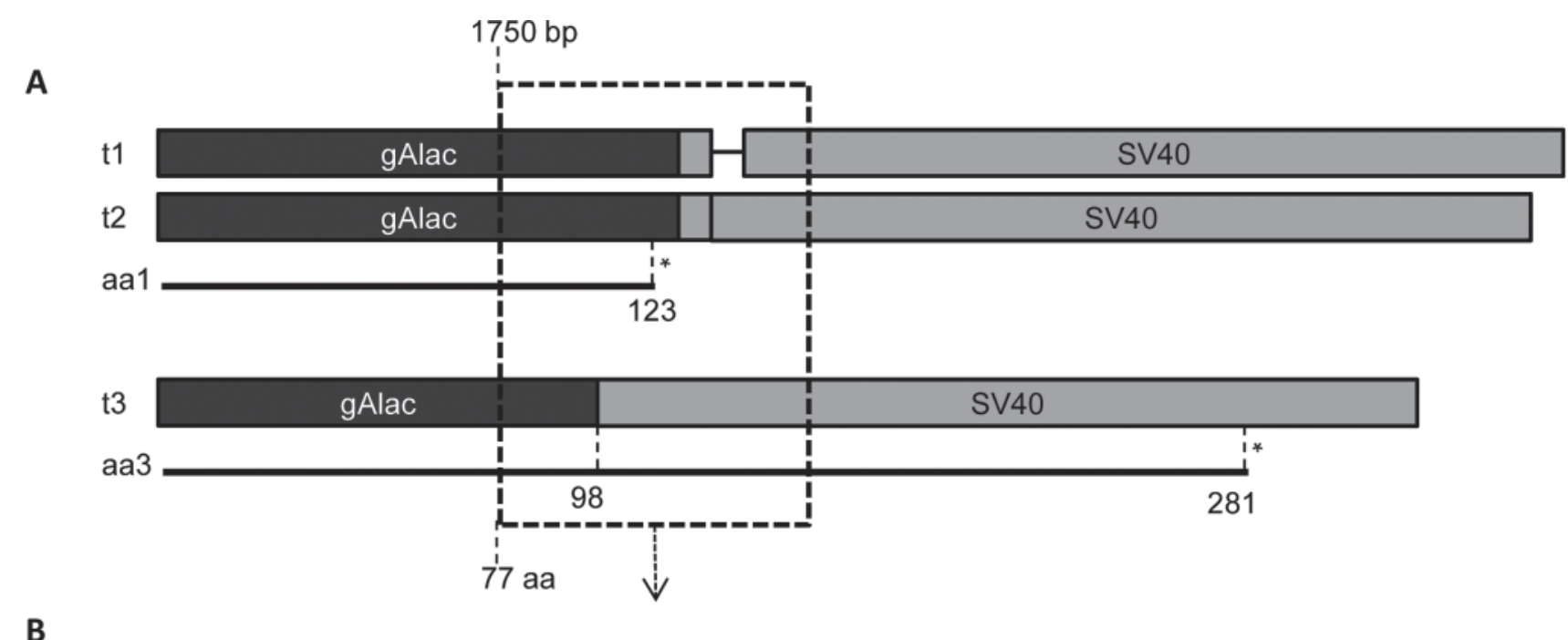

B

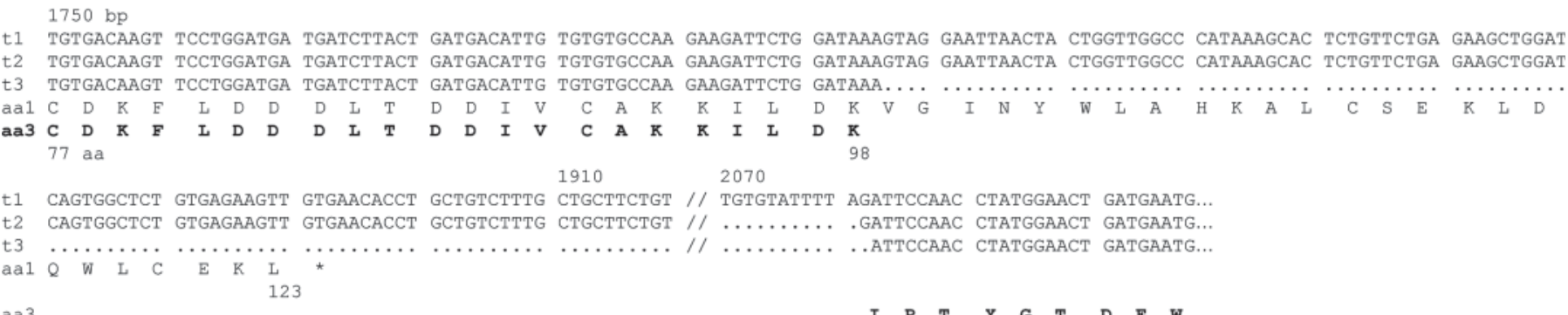

Figure 2. Alternative splicing of the goat $\alpha$-lactalbumin (gAlac) transgene. (A) Schematic representation of different splicing events observed with the gAlac minigene. Black box $=$ gAlac open reading frame $(\mathrm{ORF})$ sequence; gray box $=$ SV40 poly $(\mathrm{A})$ signal sequence; single line $=$ SV40 intronic sequence; bold lines aa1 and aa3 refer to the transcript coding sequence, starting at the first gAlac AA; asterisk = stop codon; $\mathrm{t} 1=$ unspliced transcript; $\mathrm{t} 2=$ expected spliced transcript; $\mathrm{t} 3=$ shorter unexpected transcript; aa $1=$ protein sequence of $\mathrm{gAlac}$; aa3 $=$ protein sequence encoded by the $\mathrm{t} 3$ transcript. (B) Sequence details of the 3 transcripts.

parturition (Anderson et al., 2007). An involution delay was observed $3 \mathrm{~d}$ after weaning in the 3 analyzed transgenic mice as compared with 2 nontransgenic controls (Figure 3A), evidenced by a visible decreased number of adipocytes in mammary tissues from the transgenic lines that normally replace apoptotic epithelial cells. This delay was no longer evident at involution d 6 , at which stage no apparent differences were detected visually by whole mount between transgenic and control mammary glands (Figure 3B). Sustained Alac expression at weaning affected the early involution stages, which involves local factors (Stein et al., 2007). It might result from the persistence of a lactose synthesis activity due to the abnormal regulation of the Alac transcript. The gAlac expression did not apparently hinder the following process of apoptosis.

The MMTV promoter was reportedly expressed in nonmammary secretory organs, as the endogenous virus. Assessment of the gAlac minigene pattern of expression by RT-PCR revealed that the transgene was expressed in the brain, kidney, testis, ovaries, and uterus of transgenic mice of lines 50 and 56, but not in their liver (Figure 1D). The same splicing events that were detected in the mammary gland were observed in these organs, although at different relative levels, except in the brain where the shorter transcript was not detected. Transgenic males reproduced normally (100\% of successful mating for 8 and 9 males used in lines 50 and 56 with an average litter size of $10 \pm 1$ similar to that of control FVB/N males, respectively), suggesting that gAlac expression, including that in the testicles, did not affect spermatogenesis and reproductive performances. By contrast, transgenic females were difficult to breed. Despite being mated with FVB/N males of tested fertility, $50 \%$ of transgenic adult females from both lines ( 4 out of 8 for line 50 and 3 out of 6 for line 56) showed occurrence of several successive vaginal plugs with no subsequent gestation, a phenomenon that was not observed in the 12 control FVB/N mice used in parallel that had an overall $90 \%$ frequency of pregnancy 


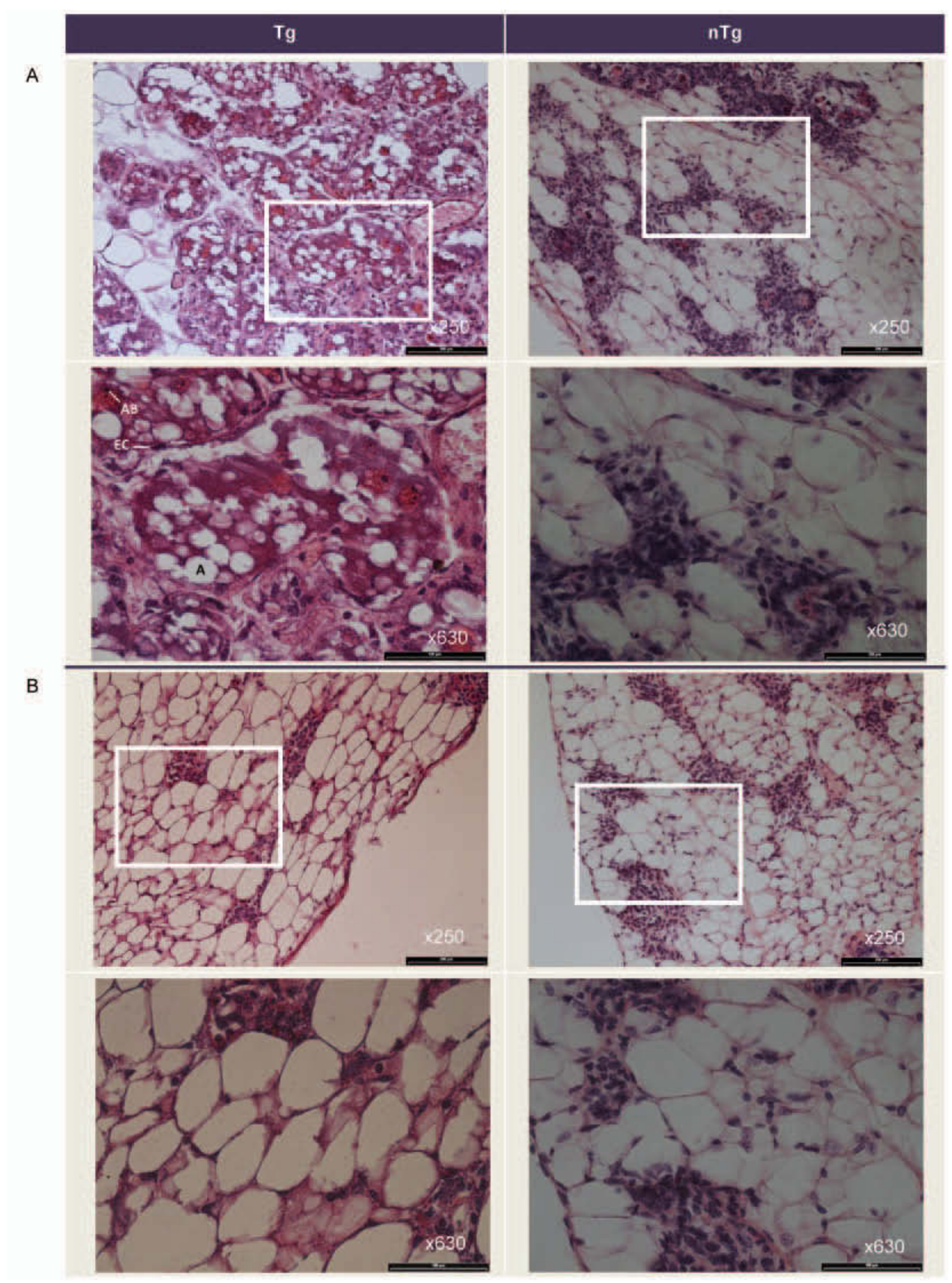

Figure 3. Early involution delay observed in transgenic mammary gland hematoxylin and eosin stained sections of mammary glands of transgenic $(\mathrm{Tg})$ and control mice $(\mathrm{nTg})$ at involution d $3(\mathrm{~A})$ and involution d $6(\mathrm{~B})$. $\mathrm{AB}=$ apoptotic body; EC = epithelial cell; $\mathrm{A}=$ adipocyte. White boxes in top panels of A and B indicate area magnified in lower panels. Color version available in the online PDF.

following vaginal plugging. The percentage of transgenic female suffering from this reproductive failure might be underestimated, as a mouse able to reproduce following one mating was classified as normal. Indeed, some of these so-called normal transgenic mice underwent a gestation after 5 to 6 failed matings that could be followed by other unsuccessful matings, suggesting that they also suffered from reproductive deficiencies. 
The duration between 2 vaginal plugs could be as short as $5 \mathrm{~d}$, suggesting the absence of pseudo-gestation in these females. The size of the litters was normal, with an average of 11 pups per dam, but the gestation length was increased in transgenic females (21 to 24 $\mathrm{d}$ versus 19 to $20 \mathrm{~d}$ in control animals). Histological analysis of ovaries of these subfertile transgenic females did not reveal any obvious alteration and transgenic females from both lines responded to superovulation treatment as efficiently as their nontransgenic controls with similar ovulation rates. The ovulated ova could be fertilized by natural mating and developed in vitro to the morula stage as efficiently as control eggs collected from nontransgenic females (with an overall rate of successful development above $80 \%$ for both types). Transgenic females were mated to vasectomized males and used as recipients for normal embryos. Six females were tested, 4 from line 50 and 2 from line 56. Sixteen 2 -cell stage embryos were transplanted per mouse and the females were killed at $13.5 \mathrm{~d}$ of gestation. Only 2 females carried implanted embryos, 1 female of line 50 with 1 implanted embryo and 1 from line 56 with 3 implanted embryos. No evidence for the occurrence of resorbing or of resorbed embryos was detected in any of the 6 analyzed females. When transferred to nontransgenic recipient mice $(\mathrm{n}=12)$, the observed pregnancy rate was above $90 \%$. Morphological analysis of the uterus did not reveal obvious phenotype differences between transgenic and control mice. Altogether, these data suggest that transgenic females from both lines had normal ovarian functions, but suffered from implantation failure.

Alac from numerous species, including gAlac have been overexpressed in the mammary gland of transgenic mice with no reported reproductive failure (Vilotte et al., 1989; Bleck and Bremel, 1994; Stacey et al., 1995). Although mammary-restricted gene expression can affect reproductive performance (Pohlmeier et al., 2010), we attributed this phenotype to the nonmammary expression of gAlac. Ectopic expression of Alac could interfere with the pituitary-ovarian axis and inhibit ovulation ( $\mathrm{Lu}$ et al., 1997).

Overall, these MMTV-gAlac transgenic mice confirmed 1) that an increase in lactating mammary Alac expression can boost the milk production, 2) that Alac deregulation during gestation has no noticeable effect on mammary gland development or differentiation or both, 3) that Alac deregulation at weaning interfered with the early involution process but had no effect on the later stages, and 4) that Alac ectopic expression altered the female reproductive capacities.

\section{ACKNOWLEDGMENTS}

We are most grateful to Fanny Joly and Mary Dutheil (both of INRA, UMR 1313 Génétique Animale et Biologie Intégrative, Jouy-en-Josas, France) for technical help.

\section{REFERENCES}

Anderson, S. M., M. C. Rudolph, J. L. McManaman, and M. C. Neville. 2007. Key stages in mammary gland development. Secretory activation in the mammary gland: It's not just about milk protein synthesis! Breast Cancer Res. 9:204.

Baltzer, A., C. Svanborg, and R. Jaggi. 2004. Apoptotic cell death in the lactating mammary gland is enhanced by a folding variant of alpha-lactalbumin. Cell. Mol. Life Sci. 61:1221-1228.

Bleck, G. T., and R. D. Bremel. 1994. Variation in expression of a bovine $\alpha$-lactalbumin transgene in milk of transgenic mice. J. Dairy Sci. 77:1897-1904.

Bleck, G. T., B. R. White, D. J. Miller, and M. B. Wheeler. 1998. Production of bovine alpha-lactalbumin in the milk of transgenic pigs. J. Anim. Sci. 76:3072-3078.

Boston, W. S., G. T. Bleck, J. C. Conroy, M. B. Wheeler, and D. J. Miller. 2001. Short communication: Effects of increased expression of alpha-lactalbumin in transgenic mice on milk yield and pup growth. J. Dairy Sci. 84:620-622.

Fujiwara, Y., R. I. Takahashi, M. Miwa, M. Kameda, K. Kodaira, M. Hirabayashi, T. Suzuki, and M. Ueda. 1999. Analysis of control elements for position-independent expression of human alpha-lactalbumin YAC. Mol. Reprod. Dev. 54:17-23.

Hakansson, A., B. Zhivotovsky, S. Orrenius, H. Sabharwal, and C. Svanborg. 1995. Apoptosis induced by a human milk protein. Proc. Natl. Acad. Sci. USA 92:8064-8068.

Lu, Q., P. Hasty, and B. D. Shur. 1997. Targeted mutation in beta1,4galactosyltransferase leads to pituitary insufficiency and neonatal lethality. Dev. Biol. 181:257-267.

McKenzie, H. A., and F. H. White Jr. 1987. Studies on a trace cell lytic activity associated with alpha-lactalbumin. Biochem. Int. $14: 347-356$.

Noble, M. S., S. Rodriguez-Zas, J. B. Cook, G. T. Bleck, W. L. Hurley, and M. B. Wheeler. 2002. Lactational performance of first-parity transgenic gilts expressing bovine alpha-lactalbumin in their milk. J. Anim. Sci. 80:1090-1096.

Pohlmeier, W. E., R. C. Hovey, and A. L. Van Eenennaam. 2010. Reproductive abnormalities in mice expressing omega-3 fatty acid desaturase in their mammary glands. Transgenic Res. doi:10.1007/ s11248-010-9407-4

Riley, L. G., P. C. Wynn, P. Williamson, and P. A. Sheehy. 2008. The role of native bovine $\alpha$-lactalbumin in bovine mammary epithelial cell apoptosis and casein expression. J. Dairy Res. 75:319-325.

Soulier, S., L. Lepourry, M. G. Stinnakre, J. C. Mercier, and J. L. Vilotte. 1997. Expression of a bovine alpha-lactalbumin transgene in alpha-lactalbumin-deficient mice can rescue lactation. In vivo relationship between bovine alpha-lactalbumin expression content and milk composition. J. Dairy Res. 64:145-148.

Stacey, A., A. Schnieke, M. Kerr, A. Scott, C. McKee, I. Cottingham, B. Binas, C. Wilde, and A. Colman. 1995. Lactation is disrupted by alpha-lactalbumin deficiency and can be restored by human alpha-lactalbumin gene replacement in mice. Proc. Natl. Acad. Sci. USA 92:2835-2839.

Stein, T., N. Salomonis, and B. A. Gusterson. 2007. Mammary gland involution as a multi-step process. J. Mammary Gland Biol. Neoplasia 12:25-35.

Stinnakre, M. G., J. L. Vilotte, S. Soulier, and J. C. Mercier. 1994 Creation and phenotypic analysis of $\alpha$-lactalbumin-deficient mice. Proc. Natl. Acad. Sci. USA 91:6544-6548. 
Svensson, M., J. Fast, A.-K. Mossberg, C. Düringer, L. Gustafsson, O. Hallgren, C. L. Brooks, L. Berliner, S. Linse, and C. Svanborg. 2003a. $\alpha$-Lactalbumin unfolding is not sufficient to cause apoptosis, but is required for the conversion to HAMLET (human $\alpha$-lactalbumin made lethal to tumor cells). Protein Sci. 12:27942804.

Svensson, M., A.-K. Mossberg, J. Pettersson, S. Linse, and C. Svanborg. 2003b. Lipids as cofactors in protein folding: Stereo-specific lipid-protein interactions are required to form HAMLET (human $\alpha$-lactalbumin made lethal to tumor cells). Protein Sci. 12:28052814

Thompson, M. P., H. M. Farrell Jr., S. Mohanam, S. Liu, W. R. Kidwell, M. P. Bansal, R. G. Cook, D. Medina, C. E. Kotts, and
M. Bano. 1992. Identification of human milk $\alpha$-lactalbumin as a cell growth inhibitor. Protoplasma 167:134-144.

Vilotte, J. L., S. Soulier, M. G. Stinnakre, M. Massoud, and J. C. Mercier. 1989. Efficient tissue-specific expression of bovine alphalactalbumin in transgenic mice. Eur. J. Biochem. 186:43-48.

Wang, J., P. Yang, B. Tang, X. Sun, R. Zhang, C. Guo, G. Gong, Y. Liu, R. Li, L. Zhang, Y. Dai, and N. Li. 2008. Expression and characterization of bioactive recombinant human alpha-lactalbumin in the milk of transgenic cloned cows. J. Dairy Sci. 91:4466-4476.

Wheeler, M. B., G. T. Bleck, and S. M. Donovan. 2001. Transgenic alteration of sow milk to improve piglet growth and health. Reprod. Suppl. 58:313-324. 